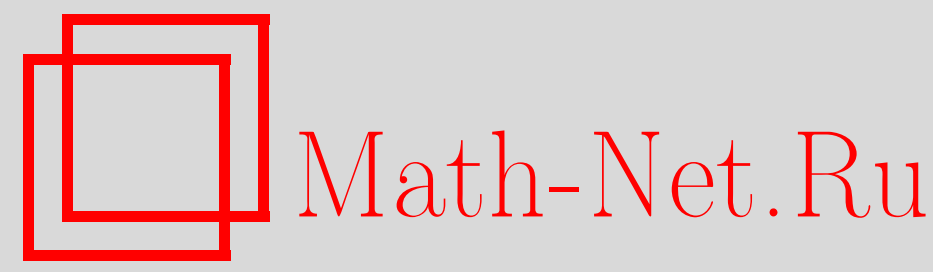

Р. М. Тригуб, Приближение функций полиномами с эрмитовской интерполяцией и ограничениями на коэффициенты, Изв. РАН. Сер. матем., 2003, том 67, выпуск 1, 199-221

DOI: https://doi.org/10.4213/im424

Использование Общероссийского математического портала Math-Net.Ru подразумевает, что вы прочитали и согласны с пользовательским соглашением

http://www.mathnet.ru/rus/agreement

Параметры загрузки:

IP : 54.196 .121 .252

26 апреля 2023 г., 10:06:14 
УДК 517.51

Р. М. Тригуб

\title{
Приближение функций полиномами с эрмитовской интерполяцией и ограничениями на коэффициенты
}

\begin{abstract}
Найден порядок приближения вещественных функций конечной гладкости многочленами с разными ограничениями: приближение функций и их производных с эрмитовской интерполяцией в концевых точках, одностороннее приближение (сверху, например), коэффициентные условия.

Библиография: 16 наименований.
\end{abstract}

1. Введение. Формулировка результатов. В настоящей статье будем изучать поточечные приближения функций с данным модулем непрерывности $r$-ой производной на отрезке вешественной оси (см. $[1$, п. 5.2$]$ или $[2$, гл. 6$])$ с разными дополнительными ограничениями.

Первое из этих ограничений - это одновременное приближение функции и ее производных полиномами с эрмитовской интерполяцией любой кратности в заданных точках (об интерполяции в концевых точках см. [3]-[5]). Эти теоремы можно, например, применить к доказательству точных достаточных условий разложения функций в ряд Фурье-Якоби (один важный пример имеется в $[6$, гл. $4, \S 5])$.

Второе ограничение - коэффициенты полиномов предполагаются цельми, при этом приходится на функцию накладывать условия арифметического характера. Критерий равномерного приближения такими полиномами предложен в [7]. Первые точные результаты о порядке приближения на отрезке $[0,1]$ (аналоги теорем Джексона и Бернштейна) получены А. О. Гельфондом [8]. Поточечные приближения на любом отрезке длины меньше четырех изучил автор [9] (см. также обзоры [10] и [11]).

Третье ограничение - это приближение функций полиномами только с одной стороны (сверху или снизу). Прямые теоремы для односторонних интегральных приближений гладких функций получены уже довольно давно, они применялись при доказательстве тауберовых теорем с остаточным членом (см., например, [12, гл. 1]).

И, наконец, недавно в связи с одной задачей о спектре положительного оператора стали изучать равномерные приближения функций полиномами с положительными коэффициентами [13] (первые прямые теоремы с дополнительными ограничениями см. в [14], [15]).

В настоящей статье делается попытка объединить все эти ограничения тогда, когда это возможно.

Приведем теоремы, которые доказываются в настоящей статье. Далее $c-$ некоторая абсолютная положительная константа, а $\gamma(a, b)$ - положительная константа, зависяшая лишь от $a$ и $b, P_{n}(W)$ - множество алгебраических полиномов степени

(C) Р.М. Тригуь, 2003 
не выше $n$ с коэффициентами из $W, \mathbb{R}_{+}=[0, \infty)=-\mathbb{R}_{-}, \mathbb{Z}_{+}=\mathbb{Z} \cap \mathbb{R}_{+}=-\mathbb{Z}_{-}$, $\omega(g ; h)$ - модуль непрерывности ограниченной функции $g$ шага $h \geqslant 0$ (см., например, [1] или [2]), $\forall x \in[a, b]$ и $n \in \mathbb{N}$

$$
\begin{gathered}
\delta_{n, a, b}(x)=\frac{1}{n} \sqrt{(x-a)(b-x)}+\frac{b-a}{2 n^{2}}, \\
\delta_{0, n, a, b}(x)=\min \left\{\frac{1}{n} \sqrt{(x-a)(b-x)}, \frac{2(x-a)(b-x)}{b-a}\right\}, \quad \delta_{n}(x)=\delta_{n,-1,1}(x) .
\end{gathered}
$$

Рассмотрим приближения функций полиномами $q_{n} \in P_{n}(\mathbb{Z})$ с эрмитовской интерполяцией. Пусть $[a, b]$ - отрезок вешественной оси длины меньше четырех, a $\left\{x_{k}\right\}_{1}^{m}$ - все целье алгебраические числа, лежащие на $[a, b]$ вместе со всеми алгебраически сопряженными к ним. Как известно (см. [7], а также [10] или [11]), существует полином с целыми коэффициентами и старшим коэффициентом, равным единице, такой, что $|X(x)|<1$ и $X(x) \neq 0$ при $x \neq x_{k}, 1 \leqslant k \leqslant m$, на $[a, b]$. Предположим еще, что $X(a)=X(b)=0$ и все нули $X$ лежат на $[a, b]$, $X_{1}(x)=\prod_{k=1}^{m}\left(x-x_{k}\right)$.

Tеорема 1. Пусть $f \in C^{r}[a, b]$ при некотором $r \in \mathbb{Z}_{+} u b-a<4$. Ecли эрмитовский интерполяционный полином $h=h_{f}$ такой, что выполнены условия: $h^{(\nu)}\left(x_{k}\right)=f^{(\nu)}\left(x_{k}\right)$ при $\nu \in[0, r] u k \in[1, m]$, имеет целье коэффициенты (это и необходимо), то для любого $n \geqslant(r+1) m-1$ существует полином $q_{n} \in P_{n}(\mathbb{Z})$ такой, что для любых $x \in[a, b] u \nu \in[0, r]$

$$
\begin{aligned}
\left|f^{(\nu)}(x)-q_{n}^{(\nu)}(x)\right| \leqslant & \gamma\left(r, X_{1}\right)\left(\min \left\{\frac{2}{b-a} \delta_{n, a, b}(x), \frac{\left|X_{1}(x)\right|}{(b-a)^{m}}\right\}\right)^{r-\nu} \\
& \times \omega\left(f^{(r)} ; \min \left\{\frac{2}{b-a} \delta_{n, a, b}(x), \frac{\left|X_{1}(x)\right|}{(b-a)^{m}}\right\}\right) .
\end{aligned}
$$

Отметим сначала, что при $X_{1}(a)=X_{1}(b)=0$ и $x \in[a, b]$

$$
\begin{gathered}
\min \left\{\frac{2}{b-a} \delta_{n, a, b}(x), \frac{\left|X_{1}(x)\right|}{(b-a)^{m}}\right\} \leqslant \min \left\{\frac{2}{b-a} \delta_{n, a, b}(x), \frac{(x-a)(b-x)}{(b-a)^{2}}\right\} \\
\leqslant 8 \min \left\{\frac{(x-a)(b-x)}{(b-a)^{2}}, \frac{\sqrt{(x-a)(b-x)}}{2(b-a) n}\right\}=\frac{4}{b-a} \delta_{0, n, a, b}(x) .
\end{gathered}
$$

Теорема 1 применима, в частности, к функциям на отрезках $[0,1],[-1,1]$, $[-\sqrt{2}, \sqrt{2}],[-1,2]$ и $[-\sqrt{3}, \sqrt{3}]$. Для последнего отрезка, например, имеем

$$
X(x)=x^{2}\left(x^{2}-1\right)\left(x^{2}-2\right)\left(x^{2}-x+1\right)\left(x^{2}+x+1\right)\left(x^{2}-3\right) .
$$

Множество отрезков в теореме 1 можно, видимо, расширить, но обязательно в предположении, что концы их - числа алгебраические.

Теперь рассмотрим односторонние приближения функций полиномами со знакопостоянными коэффициентами с эрмитовской интерполяцией в концах отрезка (сначала в интегральной норме с весом Якоби, а затем и поточечно). Ограничимся приближением сверху $\left(f(x) \leqslant p_{n}(x)\right.$ при $\left.x \in[a, b]\right)$, так как переход к приближению снизу очевиден. В силу знакопостоянства коэффициентов приближающих полиномов нужно предполагать, что $[a, b] \subset \mathbb{R}_{-}\left(\right.$при $[a, b] \subset \mathbb{R}_{+}$функция должна 
быть аналитической в окрестности нуля). Основным является случай $b=0$ (переход к общему случаю осуществляется сдвигом отрезка влево). Длина отрезка может быть любой (переход к обшему случаю осушествляется преобразованием подобия). Поэтому рассмотрим, например, отрезок $[-2,0]$.

Теорема 2. Пусть $r$ - нечетное. Если функиия $f^{(r)}$ убьвает на $[-2,0]$ от единицы до нуля, а $f^{(\nu)}(0)=0$ при $\nu \in[0, r]$, то для любого $n \geqslant 2 r+2$ существует полином в $P_{n}\left(\mathbb{Z}_{+}\right)$, а для любого $n \geqslant 2 r+3$ и полином в $P_{n}\left(\mathbb{Z}_{-}\right)$, каждый из которых удовлетворяет на $[-2,0]$ неравенствам

$$
f(x) \leqslant p_{n}(x), \quad \int_{-2}^{0} \frac{\left|f(x)-p_{n}(x)\right|}{(|x|(x+2))^{(r+1) / 2}} d x \leqslant \frac{\gamma(r)}{n^{r+1}} .
$$

Теорема 3. Пусть $r$ - нечетное. Если $f \in C^{r}[-2,0]$ u $f^{(r)}$ убьввает, а $f^{(\nu)}(0)=0$ при $\nu \in[0, r]$, то для любого $n \geqslant 2 r+2$ существует полином в $P_{n}\left(\mathbb{Z}_{+}\right)$, а для любого $n \geqslant 2 r+3$ и полином в $P_{n}\left(\mathbb{Z}_{-}\right)$, каждый из которых при $0 \leqslant \nu \leqslant r$ удовлетворяет на $[-2,0]$ неравенствам

$$
f(x) \leqslant p_{n}(x), \quad\left|f^{(\nu)}(x)-p_{n}^{(\nu)}(x)\right| \leqslant \gamma(r) \delta_{0, n,-2,0}^{r-\nu}(x) \omega\left(f^{(r)} ; \delta_{0, n,-2,0}(x)\right) .
$$

(Операторы $p_{n}=p_{n}(f)$ в теоремах 2 и 3 линейные.)

Отметим сразу, что условие $f^{(\nu)}(0)=0$ при $\nu \in[0, r]$ является необходимым. Убывание $f^{(r)}$ в теореме 3 (и теореме 2 ) нельзя заменить возрастанием (см. ниже лемму 15 ), а при четном $r$ недостаточно возрастания $f^{(r)}$ (см. там же) или убывания $f^{(r)}$ (в качестве контрпримера можно рассмотреть любую такую функцию с условием $f^{(r+1)}(0)=-\infty$; см. лемму 7$)$.

В следуюшей теореме отрезок $[-1,0]$ нельзя заменить на $[-1-\varepsilon, 0], \varepsilon>0$.

Теорема 4. Пусть $r$ - нечетное. Если $f \in C^{r}[-1,0]$ и $f^{(r)}$ убиввает и если $\frac{f^{(\nu)}(-1)}{\nu !} \in \mathbb{Z}, \quad \frac{f^{(\nu)}(0)}{\nu !} \in \mathbb{Z}_{+}$nри $\nu \in[0, r]$, то для любого $n \geqslant 2 r+2$ cуществует полином $q_{n} \in P_{n}\left(\mathbb{Z}_{+}\right)$такой, что $f(x) \leqslant q_{n}(x)$ при $x \in[-1,0]$ и при любом $\nu \in[0, r]$

$$
\left|f^{(\nu)}(x)-q_{n}^{(\nu)}(x)\right| \leqslant \gamma(r) \delta_{0, n,-1,0}^{r-\nu}(x)\left(\omega\left(f^{(r)} ; \delta_{0, n,-1,0}(x)\right)+\delta_{0, n,-1,0}(x)\right) .
$$

Для доказательства теорем 1 и 4 используются схема доказательства теоремы 1 из [9] и новые леммы $4,8,13$ и 14 (см. в п. 2). Для доказательства теорем 2 и 3 используются следуюшее очевидное представление функции на $[-2,0]$ :

$$
f(x)=\sum_{k=0}^{r} \frac{f^{(k)}(0)}{k !} x^{k}+\frac{1}{2 r !} \int_{-2}^{0}(x-y)^{r}(\operatorname{sign}(x-y)-1) d f^{(r)}(y),
$$

и теорема о приближении sign полиномами с эрмитовской интерполяцией любой кратности в концах отрезка (см. [14, теорема 1], доказательство этой теоремы, приведенной ниже в виде леммы 9 , составляет содержание статьи [16]).

Теоремы 2 и 3 анонсированы в [14].

Отметим еще, что в теоремах 2-4 найдена наименьшая из возможных степеней полиномов, а другой переход к односторонним приближениям (случай нелинейных операторов) содержится в [15]. 
2. Вспомогательные утверждения. Понадобятся некоторые усиления следующей известной теоремы А.. . Тимана (см., например, [1] или [2]). Если $f \in$ $C^{r}[-1,1]$ при некотором $r \in \mathbb{Z}_{+}$, то для любого $n \geqslant \max \{r, 1\}$ существует полином $p_{n} \in P_{n}(\mathbb{R})$ такой, что для любого $x \in[-1,1]$

$$
\left|f(x)-p_{n}(x)\right| \leqslant \gamma(r) \delta_{n}^{r}(x) \omega\left(f^{(r)} ; \delta_{n}(x)\right) .
$$

ЛЕмма 1. Из неравенства (1) следует, ито для любого $\nu \in[0, r]$ выполнено

$$
\left|f^{(\nu)}(x)-p_{n}^{(\nu)}(x)\right| \leqslant \gamma_{1}(r) \delta_{n}^{r-\nu}(x) \omega\left(f^{(r)} ; \delta_{n}(x)\right),
$$

u для тех же $x \in[-1,1]$

$$
\left|p_{n}^{(r+1)}(x)\right| \leqslant \gamma_{2}(r) \frac{\omega\left(f^{(r)} ; \delta_{n}(x)\right)}{\delta_{n}(x)} .
$$

(См. [9, лемма 1 и замечание 2], см. также, например, [2, гл. $7, \S 3]$.)

Лемма 2. Пусть $f \in C^{r}[-1,1] u f^{(\nu)}\left(x_{k}\right)=0$ nрu $\nu \in[0, r] u k \in[1, m]$, где $-1 \leqslant x_{1}<x_{2}<\cdots<x_{m} \leqslant 1$. Тогда для любого $n \geqslant n_{0}\left(r, X_{1}\right)$ существует $p_{n}$ такой, что $p_{n}^{(\nu)}\left(x_{k}\right)=0$ при $\nu \in[0, r], k \in[1, m]$ и для любого $x \in[-1,1]$

$$
\left|f(x)-p_{n}(x)\right| \leqslant \gamma_{3}(r) \delta_{n}^{r}(x) \omega\left(f^{(r)} ; \delta_{n}(x)\right) .
$$

Это утверждение см. в [9, теорема 1] (см. случаи I-III при $p_{2}(x)=1$ ).

ЛЕмма 3. 1) При $y_{k}=\sin \frac{k \pi}{2 n+1} u k \in[0, n-1]$

$$
\frac{1}{24 n} \sqrt{1-y_{k}^{2}} \leqslant y_{k+1}-y_{k} \leqslant \frac{4 \pi}{n} \sqrt{1-y_{k+1}^{2}}, \quad \delta_{n}\left(y_{k}\right) \leqslant 5 \delta_{n}\left(y_{k+1}\right) .
$$

2) Ecлu $|x-y| \leqslant c \max \left\{\delta_{n}(x), \delta_{n}(y)\right\}, m o$

$$
\max \left\{\delta_{n}(x), \delta_{n}(y)\right\} \leqslant 2(c+1) \min \left\{\delta_{n}(x), \delta_{n}(y)\right\} .
$$

3) Если $|y| \leqslant|z|, \quad|z-y| \leqslant c \delta_{n}(z), \quad \delta_{n}(y) \leqslant c \delta_{n}(z)$ при некоторых $x, y, z \in$ $[-1,1]$, mo

$$
\frac{1}{2 c+1}\left(|x-y|+\delta_{n}(y)\right) \leqslant\left(|x-y|+\delta_{n}(z)\right) \leqslant(c+1)\left(|x-y|+\delta_{n}(y)\right) .
$$

Утверждение 1) - это лемма 1 из [16], утверждение 2) - лемма 2, а утверждение 3 ) - лемма 4 из [15].

ЛЕмма 4. 1) Если полином $p_{n}$ удовлетворяет неравенству (1) и имеем $p_{n}^{(\nu)}\left(x_{k}\right)=0$ nрu $\nu \in[0, r] u k \in[1, m]$, mо при тех же $\nu$ u $x$

$$
\begin{aligned}
\left|f^{(\nu)}(x)-p_{n}^{(\nu)}(x)\right| \leqslant & \gamma_{4}(r) \min \left\{\delta_{n}(x),\left|x-x_{1}\right|, \ldots,\left|x-x_{m}\right|\right\}^{r-\nu} \\
& \times \omega\left(f^{(r)} ; \min \left\{\delta_{n}(x),\left|x-x_{1}\right|, \ldots,\left|x-x_{m}\right|\right\}\right) .
\end{aligned}
$$

2) Если при некотором $r \geqslant 0$ для любого $x \in[-1,1]$ выполнено $\left|p_{n}(x)\right| \leqslant$ $\delta_{n}^{r}(x) \omega\left(\delta_{n}(x)\right)$, где $\omega-$ функиия со свойством (4) (см. ниже), $p_{n}^{(\nu)}\left(x_{k}\right)=0$, $1 \leqslant k \leqslant m, \quad 0 \leqslant \nu \leqslant q$, при некотором $q \geqslant r$, то для любого $x \in[-1,1]$

$$
\begin{gathered}
\left|p_{n}(x)\right| \leqslant \gamma\left(r, q, X_{1}\right) \min \left\{\delta_{n}(x),\left|x-x_{1}\right|, \ldots,\left|x-x_{m}\right|\right\}^{r} \\
\times \omega\left(\min \left\{\delta_{n}(x),\left|x-x_{1}\right|, \ldots,\left|x-x_{m}\right|\right\}\right) .
\end{gathered}
$$


ДоКАЗАТЕЛЬСТВо основано на лемме 1.

1) Рассмотрим значения $x \in[-1,1]$, для которых указанньй минимум равен, например, $\left|x-x_{1}\right|$. Но тогда в силу леммы 3,2$)$

$$
\max \left\{\delta_{n}(x), \delta_{n}\left(x_{1}\right)\right\} \leqslant 4 \min \left\{\delta_{n}(x), \delta_{n}\left(x_{1}\right)\right\} .
$$

В силу формулы Тейлора существуют $y_{1}, y_{2} \in\left(x_{1}, x\right]$ такие, что

$$
\begin{aligned}
\left|f^{(\nu)}(x)-p_{n}^{(\nu)}(x)\right| & =\frac{\left|x-x_{1}\right|^{r-\nu}}{(r-\nu) !}\left|f^{(r)}\left(y_{1}\right)-p_{n}^{(r)}\left(y_{1}\right)\right| \\
& =\frac{\left|x-x_{1}\right|^{r-\nu}}{(r-\nu) !}\left|\left(f^{(r)}\left(y_{1}\right)-f^{(r)}\left(x_{1}\right)\right)+\left(p_{n}^{(r)}\left(x_{1}\right)-p_{n}^{(r)}\left(y_{1}\right)\right)\right| \\
& \leqslant \frac{\left|x-x_{1}\right|^{r-\nu}}{(r-\nu) !}\left(\omega\left(f^{(r)} ;\left|x-x_{1}\right|\right)+\left|p_{n}^{(r+1)}\left(y_{2}\right)\right|\left|x_{1}-y_{1}\right|\right) .
\end{aligned}
$$

Теперь применим лемму 1 (см. неравенство (3)). В силу известного свойства модуля непрерывности (см., например, $[1$, п. 3.2$]$ или $[2$, гл. $3, \S 1])$ для любого $\lambda>0$ и $h \in[0,2 / \lambda]$ выполнено

$$
\omega(\lambda h) \leqslant(\lambda+1) \omega(h)
$$

и (как следствие из него) при $0<h_{1} \leqslant h_{2}$

$$
\omega\left(h_{2}\right) \leqslant \omega\left(h_{1}\right)\left(\frac{h_{2}}{h_{1}}+1\right) \leqslant 2 \frac{h_{2}}{h_{1}} \omega\left(h_{1}\right) .
$$

Так как $\left|x_{1}-y_{1}\right| \leqslant\left|x-x_{1}\right|$ и $\left|x-x_{1}\right| \leqslant \delta_{n}(x) \leqslant 4 \min \left\{\delta_{n}(x), \delta_{n}\left(x_{1}\right)\right\} \leqslant 4 \delta_{n}\left(y_{2}\right)$, то

$$
\frac{\omega\left(f^{(r)} ; \delta_{n}\left(y_{2}\right)\right)}{\delta_{n}\left(y_{2}\right)} \leqslant 2 \frac{\omega\left(f^{(r)} ; \frac{\left|x-x_{1}\right|}{4}\right)}{\frac{\left|x-x_{1}\right|}{4}} \leqslant 8 \frac{\omega\left(f^{(r)} ;\left|x-x_{1}\right|\right)}{\left|x-x_{1}\right|} .
$$

2) В силу неравенства Дзядыка-Лебедя-Брудного (см., например, [1, п. 4.8.72] или $[2$, гл. $6, \S 1])$ для производной полинома выполнено неравенство

$$
\left|p_{n}^{(q+1)}(x)\right| \leqslant \gamma(r, q) \delta_{n}^{r-q-1}(x) \omega\left(\delta_{n}(x)\right) .
$$

Как и при доказательстве утверждения 1$)$, считая $m=1$ и $\left|x-x_{1}\right| \leqslant \delta_{n}(x)$, получаем при некотором $y_{1} \in\left(x_{1}, x\right)$, что

$$
\begin{aligned}
\left|p_{n}(x)\right| & =\frac{\left|x-x_{1}\right|^{q+1}}{(q+1) !}\left|p_{n}^{(q+1)}\left(y_{1}\right)\right| \leqslant \gamma_{1}(r, q)\left|x-x_{1}\right|^{q+1} \frac{\omega\left(\delta_{n}\left(y_{1}\right)\right)}{\delta_{n}\left(y_{1}\right)} \frac{1}{\delta_{n}^{q-r}\left(y_{1}\right)} \\
& \leqslant \gamma_{2}(r, q)\left|x-x_{1}\right|^{q+1} \frac{\omega\left(\left|x-x_{1}\right|\right)}{\left|x-x_{1}\right|}\left|x-x_{1}\right|^{r-q}=\gamma_{2}(r, q)\left|x-x_{1}\right|^{r} \omega\left(\left|x-x_{1}\right|\right) .
\end{aligned}
$$

Отметим, что утверждение 2) при $x_{1}=-x_{2}=1$ имеется, по сути, в [3], хотя и приведено в более слабой форме, так как

$$
\min \left\{1-x^{2}, \frac{\sqrt{1-x^{2}}}{n}+\frac{1}{n^{2}}\right\} \leqslant 2 \min \left\{1-x^{2}, \frac{\sqrt{1-x^{2}}}{n}\right\} \leqslant 2 \frac{\sqrt{1-x^{2}}}{n} .
$$




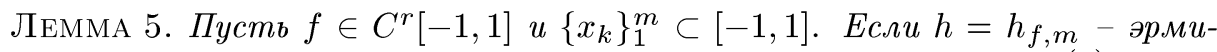
товский интерполяционный полином, определяемый условиями $h^{(\nu)}\left(x_{k}\right)=$ $f^{(\nu)}\left(x_{k}\right)$ nри $\nu \in[0, r] u k \in[1, m]\left(h \in P_{m(r+1)-1}(\mathbb{R})\right)$, mo $\left|h^{(r+1)}(x)\right| \leqslant$ $\gamma\left(r, X_{1}\right) \omega\left(f^{(r)} ; 2\right)$ и для любого $\nu \in[0, r]$

$$
\left|f^{(\nu)}(x)-h^{(\nu)}(x)\right| \leqslant \gamma\left(r, X_{1}\right) \omega\left(f^{(r)} ; \min _{k}\left|x-x_{k}\right|\right)\left(\min _{k}\left|x-x_{k}\right|\right)^{r-\nu} .
$$

ДОкАЗАТЕЛЬСТВо. При $m=1 h_{f, 1}$ - полином Тейлора степени $r$ в точке $x_{1}$ и искомые утверждения очевидны. Предположим, что лемма доказана для точек $\left\{x_{k}\right\}_{1}^{m-1}$. Представим $h_{f, m}$ в виде

$$
h_{f, m}(x)=h_{f, m-1}(x)+\left(x-x_{1}\right)^{r+1} \ldots\left(x-x_{m-1}\right)^{r+1} \sum_{k=0}^{r} a_{k}\left(x-x_{m}\right)^{k}
$$

и проверим, что

$$
\max _{1 \leqslant k \leqslant r}\left|a_{k}\right| \leqslant \gamma_{1}\left(r, X_{1}\right) \omega\left(f^{(r)} ; 2\right) .
$$

Очевидно, что при $k \in[0, r]$

$$
\begin{aligned}
\left|a_{k}\right| & =\frac{1}{k !}\left|\frac{d^{k}}{d x^{k}}\left(\frac{h_{f, m}(x)-h_{f, m-1}(x)}{\left(x-x_{1}\right)^{r+1} \ldots\left(x-x_{m-1}\right)^{r+1}}\right)_{x=x_{m}}\right| \\
& =\frac{1}{k !}\left|\frac{d^{k}}{d x^{k}}\left(\frac{f(x)-h_{f, m-1}(x)}{\left(x-x_{1}\right)^{r+1} \ldots\left(x-x_{m-1}\right)^{r+1}}\right)_{x=x_{m}}\right| \\
& \leqslant \gamma_{0}\left(r, X_{1}\right) \sum_{\nu=0}^{k}\left|f^{(\nu)}\left(x_{m}\right)-h_{f, m-1}^{(\nu)}\left(x_{m}\right)\right| .
\end{aligned}
$$

Но в силу предположения индукции

$$
\left|f^{(\nu)}(x)-h_{f, m-1}^{(\nu)}(x)\right| \leqslant \gamma\left(r,\left\{x_{k}\right\}_{1}^{m-1}\right) 2^{r-\nu} \omega\left(f^{(r)} ; 2\right) \leqslant 2^{r} \gamma \omega\left(f^{(r)} ; 2\right)
$$

и неравенство (7) установлено. Из него и предположения индукции сразу получаем

$$
\left|h_{f, m}^{(r+1)}(x)\right| \leqslant\left|h_{f, m-1}^{(r+1)}(x)\right|+\gamma_{2}\left(r, X_{1}\right) \omega\left(f^{(r)} ; 2\right) \leqslant \gamma_{3}\left(r, X_{1}\right) \omega\left(f^{(r)} ; 2\right) .
$$

Теперь для любых $x_{k}$ и $\nu \in[0, r]$ существуют $y_{1}, y_{2} \in\left(x_{k}, x\right]$ такие, что

$$
\begin{aligned}
\left|f^{(\nu)}(x)-h_{f, m}^{(\nu)}(x)\right| & =\frac{\left|x-x_{k}\right|^{r-\nu}}{(r-\nu) !}\left|f^{(r)}\left(y_{1}\right)-h_{f, m}^{(r)}\left(y_{1}\right)\right| \\
& \leqslant \frac{\left|x-x_{k}\right|^{r-\nu}}{(r-\nu) !}\left(\omega\left(f^{(r)} ;\left|x-x_{k}\right|\right)+\left|h_{f, m}^{(r+1)}\left(y_{2}\right)\right|\left|x-x_{k}\right|\right) \\
& \leqslant \gamma\left(r, X_{1}\right)\left(\omega\left(f^{(r)} ;\left|x-x_{k}\right|\right)+\omega\left(f^{(r)} ; 2\right)\left|x-x_{k}\right|\right)\left|x-x_{k}\right|^{r-\nu} .
\end{aligned}
$$

Осталось учесть, что в силу (5) при $\left|x-x_{k}\right| \leqslant 2$ имеем

$$
\omega\left(f^{(r)} ; 2\right)\left|x-x_{k}\right| \leqslant 4 \omega\left(f^{(r)} ;\left|x-x_{k}\right|\right) .
$$


ЛЕмма 6. Для любой монотонной на $[0,1]$ функции $f$ и для любых $a, b \in$ $(0,1]$

$$
\left|\int_{0}^{b} \frac{d f(y)}{\sqrt{a+y}}\right| \leqslant 5 \frac{\omega(f ; \sqrt{a b})}{\sqrt{a}} .
$$

ДоКАЗАТЕЛЬСТво. Считаем для определенности, что $f$ возрастает и $f(0)=0$. При $b \leqslant a$ в силу монотонности модуля $\omega$ имеем

$$
\int_{0}^{b} \frac{d f(y)}{\sqrt{a+y}} \leqslant \frac{1}{\sqrt{a}} \int_{0}^{b} d f(y) \leqslant \frac{1}{\sqrt{a}} \omega(f ; b) \leqslant \frac{1}{\sqrt{a}} \omega(f ; \sqrt{a b}) .
$$

Если же $a<b$, то после интегрирования по частям в интеграле Стилтьеса, учитывая, что $f(0)=0$, а оценка интеграла по $[0, a]$ уже получена, получаем

$$
\int_{a}^{b} \frac{d f(y)}{\sqrt{a+y}} \leqslant \int_{a}^{b} \frac{d f(y)}{\sqrt{y}} \leqslant \frac{f(b)}{\sqrt{b}}+\frac{1}{2} \int_{a}^{b} \frac{f(y) d y}{y^{3 / 2}} \leqslant \frac{\omega(f ; b)}{\sqrt{b}}+\frac{1}{2} \int_{a}^{b} \frac{\omega(f ; y)}{y^{3 / 2}} d y .
$$

Применяя дважды неравенство (4) при $h=\sqrt{a b}$, имеем

$$
\frac{\omega(f ; \sqrt{a b})}{\sqrt{a}}\left[\sqrt{\frac{a}{b}}\left(\sqrt{\frac{b}{a}}+1\right)+\frac{1}{2 \sqrt{a}} \int_{a}^{b} \frac{y / \sqrt{a b}+1}{y^{3 / 2}} d y\right] .
$$

Первое слагаемое в квадратной скобке меньше двух, второе слагаемое после вычисления интеграла не больше

$$
\frac{1}{2 \sqrt{a}}\left(\frac{2 \sqrt{b}}{\sqrt{a b}}+\frac{2}{\sqrt{a}}\right)=2
$$

Переходим к односторонним приближениям функций полиномами с эрмитовской интерполящией. Как видно из следующей леммы, для функции $f_{1}(x)=|x-1|^{r+1 / 2}$ вообше не сушествует полинома $p$ с условиями: $p^{(\nu)}(1)=f_{1}^{(\nu)}(1), 0 \leqslant \nu \leqslant r$, и $f_{1}(x) \leqslant p(x)$ при любом $x \in[-1,1]$. При четном $r$ для функции $f_{2}(x)=|x|^{r+1+\delta}$, $\delta \in[0,1)$, не существует полинома $p$ с условиями: $p^{(\nu)}(0)=f_{2}^{(\nu)}(0), 0 \leqslant \nu \leqslant r$, и $f_{2}(x) \leqslant p(x)$ при любом $x \in[-1,1]$.

ЛЕмма 7. Пусть $x_{1} \in[-1,1]$, a $f:[-1,1] \rightarrow \mathbb{R}$ имеет $r$ производных в точке $x_{1}$. Для того чтобы существовал полином р с условиями: $p^{(\nu)}\left(x_{1}\right)=$ $f^{(\nu)}\left(x_{1}\right), \quad 0 \leqslant \nu \leqslant r$, u $f(x) \leqslant p(x)$ при любом $x \in[-1,1]$, необходимо, а если $x_{1}=1$, или $x_{1}=-1$, или $x_{1} \in(-1,1)$ и $r$ - нечетное, то и достаточно, чтобы $f$ была ограничена сверху и

$$
\varlimsup_{x \rightarrow x_{1}}\left|x-x_{1}\right|^{-r-1}\left(f(x)-\sum_{\nu=0}^{r} \frac{f^{(\nu)}\left(x_{1}\right)}{\nu !}\left(x-x_{1}\right)^{\nu}\right)<\infty .
$$

Если при четном $r$ и $x_{1} \in(-1,1) \quad f(x) \leqslant p(x)$ при любом $x \in[-1,1]$, то и

$$
\begin{aligned}
\varlimsup_{x \rightarrow x_{1}+0}\left|x-x_{1}\right|^{-r-1}\left(f(x)-\sum_{\nu=0}^{r} \frac{f^{(\nu)}\left(x_{1}\right)}{\nu !}\left(x-x_{1}\right)^{\nu}\right) \leqslant \frac{p^{(r+1)}\left(x_{1}\right)}{(r+1) !} \\
\leqslant-\varlimsup_{x \rightarrow x_{1}-0}\left|x-x_{1}\right|^{-r-1}\left(f(x)-\sum_{\nu=0}^{r} \frac{f^{(\nu)}\left(x_{1}\right)}{\nu !}\left(x-x_{1}\right)^{\nu}\right) .
\end{aligned}
$$


ДокАЗАТЕЛЬСТво. Необходимость в общем случае очевидна, так как эти условия выполняются для любого полинома $(f=p)$.

Достаточность. Если $f$ ограничена сверху и верхний предел конечен, то существует число $M$ такое, что для любого $x \in[-1,1], x \neq x_{1}$, выполнено

$$
\left|x-x_{1}\right|^{-r-1}\left(f(x)-\sum_{\nu=0}^{r} \frac{f^{(\nu)}\left(x_{1}\right)}{\nu !}\left(x-x_{1}\right)^{\nu}\right) \leqslant M
$$

и, значит, для любого $x \neq x_{1}$

$$
f(x) \leqslant \sum_{\nu=0}^{r} \frac{f^{(\nu)}\left(x_{1}\right)}{\nu !}\left(x-x_{1}\right)^{\nu}+M\left|x-x_{1}\right|^{r+1} .
$$

Но и второе слагаемое в указанных случаях является полиномом (степени $r+1)$. Необходимость дополнительного условия при четном $r$ следует из того, что

$$
\begin{gathered}
\varlimsup_{x \rightarrow x_{1}+0}\left|x-x_{1}\right|^{-r-1}\left(f(x)-\sum_{\nu=0}^{r} \frac{f^{(\nu)}\left(x_{1}\right)}{\nu !}\left(x-x_{1}\right)^{\nu}\right) \\
\leqslant \lim _{x \rightarrow x_{1}+0} \frac{p(x)-\sum_{\nu=0}^{r} \frac{p^{(\nu)}\left(x_{1}\right)}{\nu !}\left(x-x_{1}\right)^{\nu}}{\left(x-x_{1}\right)^{r+1}}=\frac{p^{(r+1)}\left(x_{1}\right)}{(r+1) !}, \\
\varlimsup_{x \rightarrow x_{1}-0}\left|x-x_{1}\right|^{-r-1}\left(f(x)-\sum_{\nu=0}^{r} \frac{f^{(\nu)}\left(x_{1}\right)}{\nu !}\left(x-x_{1}\right)^{\nu}\right) \\
\leqslant \lim _{x \rightarrow x_{1}-0} \frac{p(x)-\sum_{\nu=0}^{r} \frac{p^{(\nu)}\left(x_{1}\right)}{\nu !}\left(x-x_{1}\right)^{\nu}}{\left|x-x_{1}\right|^{r+1}}=-\frac{p^{(r+1)}\left(x_{1}\right)}{(r+1) !} .
\end{gathered}
$$

Лемму 7 легко сформулировать для любого числа узлов интерполяции.

Будем считать далее, что имеем две точки интерполяции в конщах отрезка, и добавим условия на коэффициенты эрмитовских полиномов.

Лемма 8. Пусть $r$ - нечетное $и f^{(r)}(0) \leqslant f^{(r)}(x) \leqslant f^{(r)}(-1)$ на отрез$\kappa e[-1,0]$.

1) Если $f^{(\nu)}(0) \geqslant 0$ при $\nu \in[0, r]$, то существует полином $h \in P_{2 r+2}\left(\mathbb{R}_{+}\right)$ такой, что $f(x) \leqslant h(x)$ на отрезке $[-1,0]$ и при $\nu \in[0, r]$

$$
\left|f^{(\nu)}(x)-h^{(\nu)}(x)\right| \leqslant \gamma(r)|x(x+1)|^{r+1-\nu} \omega\left(f^{(r)} ; 1\right) .
$$

2) Если $f^{(\nu)}(0) \leqslant 0$ при $\nu \in[0, r]$, то существует полином $h \in P_{2 r+3}\left(\mathbb{Z}_{-}\right)$ такой, что на отрезке $[-1,0] f(x) \leqslant h(x)$ и при $\nu \in[0, r]$ имеет место (8).

Если в утверждениях 1) или 2) $\frac{f^{(\nu)}(0)}{\nu !}, \frac{f^{(\nu)}(-1)}{\nu !} \in \mathbb{Z}, \quad 0 \leqslant \nu \leqslant r$, то коэффичиенты $h$ можно считать еще и чельми. При этом в (8) нужно вместо $\omega\left(f^{(r)} ; 1\right)$ поставить $\omega\left(f^{(r)} ; 1\right)+1$. 
ДокАЗАТЕЛЬСтво. Отметим сразу, что степени $(2 r+2)$ и $(2 r+3)$ - наименьшие из возможных, а подобный результат справедлив и при четном $r$.

Обозначим через $h_{f}$ эрмитовский интерполяционный полином, определяемый условиями $h_{f}^{(\nu)}(-1)=f^{(\nu)}(-1)$ и $h_{f}^{(\nu)}(0)=f^{(\nu)}(0)$ при $\nu \in[0, r]$ (его степень не более $2 r+1)$. Тогда при некоторых числовых коэффициентах

$$
h_{f}(x)=\sum_{\nu=0}^{r} \frac{f^{(\nu)}(0)}{\nu !} x^{\nu}+x^{r+1} \sum_{k=0}^{r} a_{k}(x+1)^{k}=\sum_{\nu=0}^{r} \frac{f^{(\nu)}(0)}{\nu !} x^{\nu}+x^{r+1} \sum_{k=0}^{r} b_{k} x^{k} .
$$

В силу неравенства (7) имеем $\left|a_{k}\right| \leqslant \gamma_{1}(r) \omega\left(f^{(r)} ; 1\right)$, а значит, и $\left|b_{k}\right| \leqslant \gamma_{2}(r) \times$ $\omega\left(f^{(r)} ; 1\right), 0 \leqslant k \leqslant r$. Аналогично,

$$
h_{f}(x)=\sum_{\nu=0}^{r} \frac{f^{(\nu)}(-1)}{\nu !}(x+1)^{\nu}+(x+1)^{r+1} \sum_{k=0}^{r} \tilde{a}_{k} x^{k} .
$$

1) Проверим, что при $x \in[-1,0]$

$$
f(x) \leqslant h_{f}(x)+\gamma_{3}(r)|x(x+1)|^{r+1} \omega\left(f^{(r)} ; 1\right) .
$$

Пусть для определенности $x \in[-1 / 2,0]$. Сушествует $y \in(x, 0)$ такой, что

$$
\begin{aligned}
f(x)-h_{f}(x) & =\frac{x^{r+1}}{r !}\left(f^{(r)}(y)-f^{(r)}(0)\right)+\sum_{k=0}^{r} b_{k} x^{k+r+1} \leqslant \sum_{k=0}^{r} b_{k} x^{k+r+1} \\
& \leqslant x^{r+1} \sum_{k=0}^{r}\left|b_{k}\right| \leqslant(r+1) \gamma_{2}(r) x^{r+1} \omega\left(f^{(r)} ; 1\right) \\
& \leqslant \gamma_{3}(r)|x(x+1)|^{r+1} \omega\left(f^{(r)} ; 1\right) .
\end{aligned}
$$

Не всегда $b_{k} \geqslant 0$ для любого $k \in[0, r]$. Если же $b_{k}<0$, то воспользуемся неравенством

$$
b_{k} x^{k+r+1} \leqslant b_{k} x^{k+r+1}+\left|b_{k}\right| x^{r+1}(x+1)^{r+1}
$$

(справа - полином с положительными коэффициентами, а при целом $b_{k}-$ и с целыми). Имеем

$$
h_{2 r+2}(x)=h_{f}(x)+x^{r+1}(x+1)^{r+1} \sum_{k: b_{k}<0}\left|b_{k}\right|+\gamma_{3}(r) x^{r+1}(x+1)^{r+1} \omega\left(f^{(r)} ; 1\right)
$$

(при целых $b_{k}$ нужно заменить $\gamma_{3}(r) \omega\left(f^{(r)} ; 1\right)$ на $\left.\left[\gamma_{3}(r) \omega\left(f^{(r)} ; 1\right)\right]+1\right)$. Неравенство (8) следует теперь из леммы 5.

2) Теперь вместо $h_{f}$ возьмем полином $\tilde{h}_{f}$, определяемый условиями $\tilde{h}_{f}^{(\nu)}(0)=$ $f^{(\nu)}(0), \tilde{h}_{f}^{(\nu)}(-1)=f^{(\nu)}(-1)$ при $\nu \in[0, r]$ и $\tilde{h}_{f}^{(r+1)}(0)=0$ (его степень не более $2 r+2)$,

$$
\tilde{h}_{f}(x)=\sum_{\nu=0}^{r} \frac{f^{(\nu)}(0)}{\nu !} x^{\nu}+x^{r+2} \sum_{k=0}^{r} c_{k}(x+1)^{k}=\sum_{\nu=0}^{r} \frac{f^{(\nu)}(0)}{\nu !} x^{\nu}+x^{r+2} \sum_{k=0}^{r} d_{k} x^{k} .
$$


Не уменьшая обшности, можно считать, что $f^{(\nu)}(0)=0$ при $\nu \in[0, r]$. Тогда коэффициент

$$
c_{k}=\frac{1}{k !} \frac{d^{k}}{d x^{k}}\left\{x^{-r-2} \tilde{h}_{f}(x)\right\}_{x=-1}=\frac{1}{k !} \frac{d^{k}}{d x^{k}}\left\{x^{-r-2} f(x)\right\}_{x=-1}
$$

есть линейная комбинация производных $f^{(\nu)}(-1), 0 \leqslant \nu \leqslant r$. Однако при $\nu \in[0, r]$

$$
\left|f^{(\nu)}(-1)\right|=\left|f^{(\nu)}(-1)-f^{(\nu)}(0)\right| \leqslant \frac{\omega\left(f^{(r)} ; 1\right)}{(r-\nu) !} .
$$

Поэтому $\left|c_{k}\right|$, а значит, и $\left|d_{k}\right|$ не превьшают $\gamma_{4}(r) \omega\left(f^{(r)} ; 1\right)$. Как и в случае 1$)$, получаем

$$
f(x) \leqslant \tilde{h}_{f}(x)+\gamma_{5}(r)|x|^{r+2}(x+1)^{r+1} \omega\left(f^{(r)} ; 1\right) .
$$

Но теперь к $\tilde{h}_{f}$ добавляется полином с отрицательными коэффициентами, а если $d_{k}>0$, то воспользуемся неравенством

$$
d_{k} x^{k+r+2} \leqslant d_{k} x^{k+r+2}+d_{k}|x|^{r+2}(x+1)^{r+1} .
$$

Положим теперь для любых $x, y \in[-1,1], n \in \mathbb{N}$ и $s, t, r \in \mathbb{R}_{+}$

$$
\begin{aligned}
\Delta_{n}(x, y, s, t, r)= & \left(\frac{1-x^{2}}{1-x^{2}+1-y^{2}+n^{-2}|\operatorname{sign} x-\operatorname{sign} y|}\right)^{s} \\
& \times\left(\frac{\delta_{n}(y)}{|x-y|+\delta_{n}(y)}\right)^{t}|x-y|^{r} .
\end{aligned}
$$

Очевидно, что $\Delta_{n}$ как функция от $(x, y)$ симметрична относительно $(0,0)$, убывает с ростом $s$ и $t$ и почти убывает с ростом $r$.

Лемма 9. Для любых $s, t \in \mathbb{R}_{+}$, любого $y \in(-1,1)$ u $n>2 s$ существует полином $p_{n}=p_{n, y}$ по $x$ степени не выше $n$, удовлетворяющий на отрезке $[-1,1]$ неравенству

$$
\left|\operatorname{sign}(x-y)-p_{n}(x)\right| \leqslant \gamma(s, t) \Delta_{n}(x, y, s, t, 0) .
$$

При этом полином $p_{n}$ на $[-1,1]$ возрастает от -1 до 1 , а все нули производной $p_{n}^{\prime}$ лежат на $[-1,1]$. Степень полинома $p_{n}$ нечетная, а старший коэффициент можно считать положительным или отрицательным. Коэффичиенты $p_{n}$ как функиии от у кусочно непрерывные.

(См. [14], доказательство этой леммы составляет содержание статьи [16].)

Лемма 10. Для любых $x, y \in[-1,1]$ u $n \in \mathbb{N}$ выполнено неравенство

$$
\begin{aligned}
& \left(\frac{1-x^{2}}{1-x^{2}+1-y^{2}+n^{-2}|\operatorname{sign} x-\operatorname{sign} y|}\right)^{1 / 2} \times \\
& \quad \times \frac{\delta_{n}(x)+\delta_{n}(y)}{|x-y|+\delta_{n}(x)+\delta_{n}(y)}|x-y| \leqslant 3 \frac{\sqrt{1-x^{2}}}{n} .
\end{aligned}
$$


ДоказАТЕльСтво. Пусть сначала $x y<0$. Учитьвая вид $\delta_{n}(x)$ (см. п. 1 ), перепишем левую часть искомого неравенства следующим образом:

$$
\frac{\sqrt{1-x^{2}}}{n}\left(\frac{\sqrt{1-x^{2}}+\sqrt{1-y^{2}}+2 n^{-1}}{\left(1-x^{2}+1-y^{2}+2 n^{-2}\right)^{1 / 2}}\right)\left(\frac{|x-y|}{|x-y|+\delta_{n}(x)+\delta_{n}(y)}\right) .
$$

Последний из трех множителей не больше единицы.

Используя в знаменателе неравенство

$$
\left(\frac{a^{2}+b^{2}+c^{2}}{3}\right)^{1 / 2} \geqslant \frac{a+b+c}{3}
$$

получаем

$$
\begin{aligned}
\left(1-x^{2}+1-y^{2}+2 n^{-2}\right)^{1 / 2} & \geqslant \frac{1}{\sqrt{3}}\left(\sqrt{1-x^{2}}+\sqrt{1-y^{2}}+\sqrt{2} n^{-1}\right) \\
& \geqslant \frac{1}{\sqrt{6}}\left(\sqrt{1-x^{2}}+\sqrt{1-y^{2}}+2 n^{-1}\right),
\end{aligned}
$$

и искомое неравенство доказано с константой $\sqrt{6}<3$.

Пусть теперь $x y \geqslant 0$. Используя неравенства $\delta_{n}(x) \geqslant \frac{1}{n^{2}}$ и $|x-y| \leqslant 1-x^{2}+$ $1-y^{2}$, получаем

$$
\begin{aligned}
\frac{\sqrt{1-x^{2}}}{n} & \frac{1}{\left(1-x^{2}+1-y^{2}\right)^{1 / 2}}\left(\sqrt{1-x^{2}}+\sqrt{1-y^{2}}+2 n^{-1}\right) \frac{|x-y|}{|x-y|+2 n^{-2}} \\
\leqslant & \frac{\sqrt{1-x^{2}}}{n} \frac{1}{\left(1-x^{2}+1-y^{2}\right)^{1 / 2}}\left(\sqrt{1-x^{2}}+\sqrt{1-y^{2}}+2 n^{-1}\right) \\
& \times \frac{1-x^{2}+1-y^{2}}{1-x^{2}+1-y^{2}+2 n^{-2}} \\
= & \frac{\sqrt{1-x^{2}}}{n} \frac{\left(1-x^{2}+1-y^{2}\right)^{1 / 2}\left(\sqrt{1-x^{2}}+\sqrt{1-y^{2}}+2 n^{-1}\right)}{1-x^{2}+1-y^{2}+2 n^{-2}} .
\end{aligned}
$$

Рассмотрим функцию

$$
g\left(x_{1}, x_{2}, x_{3}\right)=\frac{\left(x_{1}^{2}+x_{2}^{2}\right)^{1 / 2}\left(x_{1}+x_{2}+\sqrt{2} x_{3}\right)}{x_{1}^{2}+x_{2}^{2}+x_{3}^{2}}
$$

где $x_{1}=\sqrt{1-x^{2}}, x_{2}=\sqrt{1-y^{2}}$ и $x_{3}=\frac{\sqrt{2}}{n}$. Но эта функция однородная нулевой степени, а на единичной сфере выполнено $x_{1}^{2}+x_{2}^{2}+x_{3}^{2}=1$, значит,

$$
x_{1}+x_{2} \leqslant \sqrt{2}\left(x_{1}^{2}+x_{2}^{2}\right)^{1 / 2} \leqslant \sqrt{2}, \quad x_{3} \leqslant 1 .
$$

Поэтому $g\left(x_{1}, x_{2}, x_{3}\right) \leqslant \sqrt{2}+\sqrt{2}=2 \sqrt{2}<3$.

ЛЕмма 11. Для любого $y \in[-1,1] u n \in \mathbb{N}$

$$
\int_{-1}^{1} \frac{\delta_{n}^{2}(x)+\delta_{n}^{2}(y)}{(x-y)^{2}+\delta_{n}^{2}(x)+\delta_{n}^{2}(y)}\left(1-x^{2}\right)^{-1 / 2} d x \leqslant \frac{c}{n} .
$$


ДоказАтельСтво. Считаем далее, что $y \in[0,1]$ (случай $y \in[-1,0]$ сводится к рассматриваемому заменой в интеграле $x$ на $-x$ ). Интеграл разобьем на два: по $[-1,0]$ и $[0,1]$. Первый из них не больше второго, так как при $x \leqslant 0$ и $y \in[0,1]$ имеем $|x+y| \leqslant|x-y|$. Остается рассмотреть интеграл по $[0,1]$. Очевидно, что при $x \in[0,1]$ имеем $1-x \leqslant 1-x^{2} \leqslant 2(1-x)$. Поэтому

$$
\begin{aligned}
\delta_{n}^{2}(x)+\delta_{n}^{2}(y) & =\left(\left(1-x^{2}\right)^{1 / 2} \frac{1}{n}+\frac{1}{n^{2}}\right)^{2}+\left(\left(1-y^{2}\right)^{1 / 2} \frac{1}{n}+\frac{1}{n^{2}}\right)^{2} \\
& \leqslant 4\left(\frac{1-x}{n^{2}}+\frac{1-y}{n^{2}}+\frac{1}{n^{4}}\right) \leqslant 4\left(\frac{2(1-y)}{n^{2}}+\frac{|x-y|}{n^{2}}+\frac{1}{n^{4}}\right) .
\end{aligned}
$$

В случаях $1 / n^{2} \leqslant 1-y$ и $1-y \leqslant 1 / n^{2}$ из трех слагаемых остаются два. Но функция $\phi(t)=t /(a+t)$, где $a>0$, возрастает и полуаддитивна на $[0, \infty)\left(\phi\left(t_{1}+t_{2}\right) \leqslant\right.$ $\left.\phi\left(t_{1}\right)+\phi\left(t_{2}\right)\right)$. Таким образом, достаточно доказать, что каждый из следующих трех интегралов является $O(1 / n)$ равномерно по $y$ (последний - в предположении $\left.1-y \leqslant 1 / n^{2}\right)$ :

$$
\begin{gathered}
\int_{0}^{1} \frac{\left((1-y) / n^{2}\right)(1-x)^{-1 / 2}}{(x-y)^{2}+(1-y) / n^{2}} d x, \quad \int_{0}^{1} \frac{\left(|x-y| / n^{2}\right)(1-x)^{-1 / 2}}{(x-y)^{2}+|x-y| / n^{2}} d x \\
\int_{0}^{1} \frac{\left(1 / n^{4}\right)(1-x)^{-1 / 2}}{(x-y)^{2}+1 / n^{4}} d x .
\end{gathered}
$$

Первый интеграл разобьем на два. В одном из них $0 \leqslant x \leqslant(1+y) / 2$ и, значит, $1-x \geqslant(1-y) / 2$, а в другом $(1+y) / 2 \leqslant x \leqslant 1$ и, значит, $x-y \geqslant(1-y) / 2$. Имеем

$$
\begin{aligned}
& \int_{0}^{(1+y) / 2} \frac{(1-y)^{1 / 2}}{n^{2}(x-y)^{2}+1} d x+\int_{(1+y) / 2}^{1} \frac{(1-x)^{-1 / 2}}{n^{2}(1-y) / 4+1} d x \\
& =\frac{\sqrt{1-y}}{n}[\operatorname{arctg} n(x-y)]_{0}^{(1+y) / 2}+\frac{\sqrt{2}}{n} \frac{2 \sqrt{n^{2}(1-y) / 4}}{n^{2}(1-y) / 4+1} \leqslant \frac{\pi+\sqrt{2}}{n} .
\end{aligned}
$$

И второй интеграл разбиваем на два. В одном из них $|x-y| \geqslant 1-x$, а в другом $1-x \geqslant|x-y|$. Получаем

$$
\begin{aligned}
\int_{0}^{1} \frac{(1-x)^{-1 / 2}}{n^{2}|x-y|+1} d x & \leqslant \int_{0}^{1} \frac{(1-x)^{-1 / 2}}{n^{2}(1-x)+1} d x+\int_{0}^{1} \frac{|x-y|^{-1 / 2}}{n^{2}|x-y|+1} d x \\
& \leqslant 2 \int_{-\infty}^{\infty} \frac{\sqrt{|t|}}{n^{2}|t|+1} d t=\frac{2 \pi}{n}
\end{aligned}
$$

При $n \geqslant 2$ и третий интеграл разбиваем на два: по [0,1 $\left.-2 / n^{2}\right]$ и $\left[1-2 / n^{2}, 1\right]$. Оставляя в знаменателе каждого из них одно слагаемое из двух и учитывая при этом, что в первом $y-x \geqslant 1-1 / n^{2}-x \geqslant(1-x) / 2$ при $y \in\left[1-1 / n^{2}, 1\right]$, получаем

$$
\int_{0}^{1-2 / n^{2}} \frac{(1-x)^{-1 / 2}}{n^{4}(1-x)^{2} / 4} d x+\int_{1-2 / n^{2}}^{1}(1-x)^{-1 / 2} d x=O\left(\frac{1}{n}\right) .
$$


ЛЕмма 12. При $y_{k}=\sin \frac{k \pi}{2 n+1} u k \in[-n, n-1]$ (определение $\Delta_{n}(x, y, s, t, r)$ см. в (9)) выполнено неравенство

$$
\left(y_{k+1}-y_{k}\right) \max _{y_{k} \leqslant y \leqslant y_{k+1}} \Delta_{n}(x, y, s, t, r) \leqslant \gamma(s, t, r) \int_{y_{k}}^{y_{k+1}} \Delta_{n}(x, y, s, t, r) d y .
$$

ДокАЗАТЕЛЬСТво. Очевидно, что $\Delta_{n}(x, y, s, t, r)=\Delta_{n}(x, y, s, t, 0)|x-y|^{r}$. Докажем сначала, что

$$
\max _{y_{k} \leqslant y \leqslant y_{k+1}} \Delta_{n}(x, y, s, t, 0) \leqslant c^{s+t} \min _{y_{k} \leqslant y \leqslant y_{k+1}} \Delta_{n}(x, y, s, t, 0) .
$$

В силу симметрии функции $\Delta_{n}$ можно ограничиться рассмотрением случая $0 \leqslant$ $y_{k} \leqslant y \leqslant y_{k+1}$. Очевидно, что можно ограничиться значениями $s=1, t=0$ и $s=0, t=1$, т. е. доказать только то, что отношение максимума выражения в одной из круглых скобок в (9) к его минимуму ограничено абсолютной константой.

Для дроби в первой скобке выражения (9) имеем

$$
\frac{1-x^{2}+1-y_{k}^{2}+n^{-2}|\operatorname{sign} x-\operatorname{sign} y|}{1-x^{2}+1-y_{k+1}^{2}+n^{-2}|\operatorname{sign} x-\operatorname{sign} y|} \leqslant \frac{1-y_{k}^{2}}{1-y_{k+1}^{2}},
$$

и нужно воспользоваться леммой 3,1$)$. K выражению во второй скобке применим лемму 3,3$)$ при $y \in\left[y_{k}, y_{k+1}\right]$ и $z=y_{k+1}$. Получим при $c=4 \pi$ для любого $x \in[-1,1]$

$$
\frac{1}{2 c+1}\left(|x-y|+\delta_{n}(y)\right) \leqslant\left|x-y_{k+1}\right|+\delta_{n}\left(y_{k+1}\right) \leqslant(c+1)\left(|x-y|+\delta_{n}(y)\right) .
$$

Остается доказать, что для любого $r \in \mathbb{R}_{+}$и любого $x \in[-1,1]$ выполнено неравенство

$$
\gamma(r)\left(y_{k+1}-y_{k}\right) \max _{y_{k} \leqslant y \leqslant y_{k+1}}|x-y|^{r} \leqslant \int_{y_{k}}^{y_{k+1}}|x-y|^{r} d y .
$$

Пусть сначала $x \notin\left[y_{k}, y_{k+1}\right]$. Тогда

$$
\int_{y_{k}}^{y_{k+1}}|x-y|^{r} d y=\frac{1}{r+1}|| x-\left.y_{k}\right|^{r+1}-\left|x-y_{k+1}\right|^{r+1} \mid \text {. }
$$

Но при $0 \leqslant a \leqslant b$ и $r \in \mathbb{R}_{+}$

так что

$$
\frac{1}{2}(b-a)\left(a^{r}+b^{r}\right) \leqslant(b-a) b^{r} \leqslant b^{r+1}-a^{r+1},
$$

$$
\begin{aligned}
\int_{y_{k}}^{y_{k+1}}|x-y|^{r} d y & \geqslant \frac{y_{k+1}-y_{k}}{2(r+1)}\left(\left|x-y_{k}\right|^{r}+\left|x-y_{k+1}\right|^{r}\right) \\
& \geqslant \frac{y_{k+1}-y_{k}}{2(r+1)} \max _{y_{k} \leqslant y \leqslant y_{k+1}}|x-y|^{r} .
\end{aligned}
$$

Пусть теперь $x \in\left[y_{k}, y_{k+1}\right]$. Считаем для определенности, что $x \in\left[z_{k}, y_{k+1}\right]$, где $z_{k}=\frac{1}{2}\left(y_{k}+y_{k+1}\right)$. В силу предыдущего

$$
\int_{y_{k}}^{y_{k+1}}|x-y|^{r} d y \geqslant \int_{y_{k}}^{z_{k}}|x-y|^{r} d y \geqslant \frac{\left(z_{k}-y_{k}\right)}{2(r+1)}\left(\left|x-y_{k}\right|^{r}+\left|x-z_{k}\right|^{r}\right) .
$$

Но $z_{k}-y_{k}=\frac{1}{2}\left(y_{k+1}-y_{k}\right)$, a $\max |x-y|=\left|x-y_{k}\right|$. Поэтому и в этом случае

$$
\int_{y_{k}}^{y_{k+1}}|x-y|^{r} d y \geqslant \frac{y_{k+1}-y_{k}}{4(r+1)} \max _{y_{k} \leqslant y \leqslant y_{k+1}}|x-y|^{r} .
$$


ЛЕмма 13. Для любых $k, m \in \mathbb{N}$ существует постоянная $\gamma(k, m)$ такая, что для любого $n \in \mathbb{N}$ существует полином $p_{n}$, удовлетворяющий на $[0,1]$ неравенству

$$
0 \leqslant x^{k}-x^{k+m} p_{n}(x) \leqslant \gamma(k, m) \min \left\{\frac{1}{n^{2}}, x\right\}^{k} .
$$

ДоКАЗАТЕЛЬСТво. Достаточно рассмотреть случай $k=1$, а затем возвести это неравенство в степень $k \geqslant 2$.

При $1 \leqslant n \leqslant m$ можно взять $p_{n}=1$. Считая далее $n \geqslant m+1$, применим лемму $2^{\prime \prime}$ из [9]: сушествует полином $p_{n-m}$ такой, что для любого $x \in[0,1]$ вьполнено неравенство

$$
\left|x-x^{m+1} p_{n-m}(x)\right| \leqslant \frac{\gamma_{1}(m)}{n^{2}} .
$$

Возводя это неравенство в квадрат и разделив на $x$, получим утверждение леммы при $k=1$. Действительно, к полиному

$$
s(x)=s_{2 n-m-1}(x)=\left(x-x^{m+1} p_{n-m}(x)\right)^{2}
$$

с условиями

$$
|s(x)| \leqslant \frac{\gamma_{1}^{2}}{n^{4}}, \quad s(0)=s^{\prime}(0)=0,
$$

применяем лемму 4,2 ) на отрезке $[0,1]$ (при $x_{1}=r=0, q=1, \omega(h)=1$ ). Тогда

И

$$
|s(x)| \leqslant 4 \gamma_{1}^{2} \min \left\{x^{2}, \frac{1}{n^{4}}\right\}\left(\frac{2 n-m-1}{n}\right)^{4} \leqslant 64 \gamma_{1}^{2} \min \left\{x^{2}, \frac{1}{n^{4}}\right\}
$$

$$
0 \leqslant \frac{s(x)}{x} \leqslant \frac{64 \gamma_{1}^{2}}{x} \min \left\{x^{2}, \frac{1}{n^{4}}\right\} \leqslant 64 \gamma_{1}^{2} \min \left\{x, \frac{1}{n^{2}}\right\} .
$$

Лемма 14. Для любого $n \in \mathbb{N}$ существует полином $q_{n}^{+} \in P_{n}\left(\mathbb{Z}_{+}\right)$такой, что при $x \in[-1,0]$

$$
0 \leqslant-x-x^{2} q_{n}^{+}(x) \leqslant c \min \left\{\frac{1}{n^{2}},|x(x+1)|\right\} .
$$

При этом до степени $[n / 2]$ коэффичиенты $q_{n}$ не равны нулю.

ДокАЗАТЕльСтво. Пусть $2^{s} \leqslant n<2^{s+1}$ при $s \in \mathbb{Z}_{+}$. Если полином Чебьшева равен $T_{n}(x)=\cos n \arccos x$ на $[-1,1]$, то искомый полином равен

$$
\frac{T_{2^{s}}(1)-T_{2^{s}}(2 x+1)}{2 T_{2^{s}}^{\prime}(1)}=-x-\cdots
$$

При любом $k \in\left[1,2^{s}\right]$ коэффициент при $x^{k}$ равен $-2^{k-1} \frac{T_{2^{s}}^{(k)}(1)}{T_{2^{s}}^{\prime}(1)}$. То, что эти числа целые, легко доказать индукцией по $s$, если заметить, что в силу формулы двойного угла для косинуса $T_{2 n}(x)=2 T_{n}^{2}(x)-1$.

Далее очевидно, что $\left|T_{n}(x)\right| \leqslant 1=T_{n}(1)$, а максимум модуля указанного полинома на $[-1,0]$ равен $\left(T_{2^{s}}^{\prime}(1)\right)^{-1}=4^{-s}<4 n^{-2}$. Поскольку этот полином равен нулю при $x=-1$ и $x=0$, то, как и при доказательстве леммы 13 , получаем нужную оценку $(c=4)$. 
Лемма 15. 1) Пусть $r \in \mathbb{Z}_{+}$. Для любых $p \in(0,+\infty] u n \geqslant r+1$ имеем

$$
\sup \left\|f^{(r)}\right\|_{\infty}^{-1} \inf _{\substack{p_{n}: p_{n}(x) \geqslant f(x) \\(-1)^{(\nu)} f^{(\nu)}(1) \geqslant(-1)^{(\nu)} p^{(\nu)}(1) \\ 0 \leqslant \nu \leqslant r}}\left(\int_{-1}^{1}\left|f(x)-p_{n}(x)\right|^{p} d x\right)^{1 / p}=+\infty .
$$

Здесь верхняя грань берется по всем функциям $f \in C^{r}[-1,1]$, у которых $(-1)^{\nu} f^{(\nu)}$ при $\nu \in[0, r]$ убъьвают.

2) Для любого $r \in \mathbb{Z}_{+} u n \geqslant r+1$

$$
\sup \left\|f^{(r)}\right\|_{\infty}^{-1} \inf _{\substack{p_{n}: p_{n}(x) \geqslant f(x) \\ f^{(\nu)}(1)=p^{(\nu)}(1) \\ 0 \leqslant \nu \leqslant r}}\left\|f-p_{n}\right\|_{\infty}=+\infty .
$$

Здесь верхняя грань берется по всем функииям $f \in C^{r}[-1,1]$, у которых $(-1)^{r} f^{(r)}$ убиввает на $[-1,1]$.

Утверждение 2) является очевидным следствием 1$)\left(\|f\|_{\infty}\right.$ - sup-норма).

Положим при $\varepsilon \in(0,1)$

$$
f_{y}(x)=|y-x|^{\varepsilon}(\max \{y-x, 0\})^{r} .
$$

Так как $f_{y}^{(r+1)}(1)=0$ при $y \in[-1,1)$, то в силу леммы 7 для любого $n \geqslant r+1$ существует полином $p_{n}$ такой, что $f(x) \leqslant p_{n}(x)$ для любого $x \in[-1,1]$. Кроме того,

$$
f_{y}^{(r)}(x)=(r+\varepsilon)(r+\varepsilon-1) \ldots(1+\varepsilon)(\max \{y-x, 0\})^{\varepsilon}(-1)^{r}
$$

и, значит, $\left\|f_{y}^{(r)}\right\|_{\infty}=(r+\varepsilon)(r+\varepsilon-1) \ldots(1+\varepsilon)(1+y)^{\varepsilon} \leqslant(r+1) ! 2^{\varepsilon}$ при $y \in[0,1)$, a $\left\|f_{y}\right\|_{p} \leqslant\left\|f_{y}\right\|_{\infty} \leqslant 2^{r+\varepsilon}$. Предположим теперь, что существуют $b \in \mathbb{R}, n \geqslant r+1$ и $p \in(0,+\infty]$ такие, что для любого $y \in[0,1)$ найдется полином $p_{n}=p_{n, y}$ такой, что

$$
\int_{-1}^{1}\left|f_{y}-p_{n, y}\right|^{p} d x \leqslant b^{p}, \quad f_{y}(x) \leqslant p_{n, y}(x), \quad(-1)^{\nu} p_{n, y}^{(\nu)}(1) \leqslant(-1)^{\nu} f_{y}^{(\nu)}(1)=0
$$

при $\nu \in[0, r]$. В силу неравенства Гёльдера можно ограничиться случаем $p \in$ $(0,1]$. В силу неравенства треугольника имеем

$$
\int_{-1}^{1}\left|p_{n, y}(x)\right|^{p} d x \leqslant b^{p}+\int_{-1}^{1}\left|f_{y}(x)\right|^{p} d x \leqslant b^{p}+2^{(r+\varepsilon) p+1} .
$$

Применяя неравенство А. А. Маркова и неравенство разных метрик (см., например, $[1$, п. 4.8.7]), получаем

$$
\left\|p_{n}^{(r+1)}\right\|_{\infty} \leqslant n^{2(r+1)}\left\|p_{n}\right\|_{\infty} \leqslant n^{2(r+1)+2 / p}\left(b_{p}+2^{(r+\varepsilon) p+1}\right)^{1 / p} \leqslant \gamma(p, n, r, b) .
$$

Используя формулу Тейлора, теперь имеем

$$
\begin{aligned}
(1-y)^{r+\varepsilon} & =f_{y}(2 y-1)-f_{y}(1) \leqslant p_{n}(2 y-1)-p_{n}(1) \\
& =\sum_{\nu=1}^{r} \frac{(-1)^{\nu} p_{n}^{(\nu)}(1)}{\nu !}(1-y)^{\nu} 2^{\nu}+\frac{p_{n}^{(r+1)}(\theta)}{(r+1) !}(2 y-2)^{r+1} \\
& \leqslant \frac{p_{n}^{(r+1)}(\theta)}{(r+1) !} 2^{r+1}(y-1)^{r+1} \leqslant \frac{\gamma(p, n, r, b)}{(r+1) !} 2^{r+1}(1-y)^{r+1} .
\end{aligned}
$$

При $y \rightarrow 1-0$ получаем противоречие с предположением. 


\section{3. Доказательства теорем 1-4.}

ДОКАЗАТЕЛЬСТВо ТЕОРЕМЫ 1 . В силу леммы 2 для любого $n \geqslant n_{0}\left(r, X_{1}\right)$ существует полином $p_{n}$ такой, что $p_{n}^{(\nu)}\left(x_{k}\right)=f^{(\nu)}\left(x_{k}\right), 0 \leqslant \nu \leqslant r, 1 \leqslant k \leqslant m$, и на $[a, b]$ выполнено неравенство

$$
\left|f(x)-h_{f}(x)-p_{n}(x)\right| \leqslant \gamma\left(r, X_{1}\right) \delta_{n, a, b}^{r}(x) \omega\left(f^{(r)}-h_{f}^{(r)} ; \delta_{n, a, b}(x)\right),
$$

а в силу леммы 5 и соотношения $(5)$ при $h \in[0, b-a]$ выполнено

$$
\begin{aligned}
\omega\left(f^{(r)}-h_{f}^{(r)} ; h\right) \leqslant & \omega\left(f^{(r)} ; h\right)+\left\|h_{f}^{(r+1)}\right\|_{\infty} h \leqslant \omega\left(f^{(r)} ; h\right) \\
& +\gamma_{1}\left(r, X_{1}\right) \frac{2}{b-a} \omega\left(f^{(r)} ; b-a\right) h \leqslant\left(1+\gamma_{1}\right) \omega\left(f^{(r)} ; h\right) .
\end{aligned}
$$

Так как полином $p_{n}$ делится без остатка на $X_{1}^{r+1}$, то на основании леммы 13 с заменой $x$ на $X_{1}$ приближаем его полиномом с целыми коэффициентами и полиномом, делящимся уже на $X_{1}^{2 r+2}$. Затем последний из них на основании леммы 3 из [9] приближаем полиномом с целыми коэффициентами (см. [9] или ниже доказательство теоремы 4). Получаем

$$
\left|f(x)-h_{f}(x)-q_{n}(x)\right| \leqslant \gamma_{2}\left(r, X_{1}\right)\left(\delta_{n, a, b}^{r}(x) \omega\left(f^{(r)} ; \delta_{n, a, b}(x)\right)+\frac{(b-a)^{r+1}}{n^{2 r+2}}\right) .
$$

При этом $h_{f}^{(\nu)}\left(x_{k}\right)+q_{n}^{(\nu)}\left(x_{k}\right)=f^{(\nu)}\left(x_{k}\right), 0 \leqslant \nu \leqslant r, 1 \leqslant k \leqslant m$. Применяем лемму 4 , и теорема 1 доказана для $n \geqslant n_{1}\left(r, X_{1}\right)$. При $(r+1) m-1 \leqslant n<n_{1}\left(r, X_{1}\right)$ в качестве искомого полинома приближения можно взять просто $h_{f}$ (см. лемму 5 ).

ДокАЗАТЕЛЬСтво тЕоремы 2. Заменим отрезок $[-2,0]$ на $[-1,1]$ (сдвиг на единицу). Теперь по условию имеем $f^{(\nu)}(1)=0,0 \leqslant \nu \leqslant r$, нужно построить два полинома $p_{n}$ с условиями $p_{n}^{(\nu)}(1) \geqslant 0$ и $p_{n}^{(\nu)}(1) \leqslant 0$ для любого $\nu \in \mathbb{Z}_{+}$. Очевидно, что

$$
f(x)=\frac{(-1)^{r+1}}{r !} \int_{x}^{1}(y-x)^{r} d f^{(r)}(y)=\int_{-1}^{1} h_{r, y}(x) d f^{(r)}(y)
$$

где $h_{r, y}(x)=\frac{(x-y)^{r}}{2 r !}(\operatorname{sign}(x-y)-1), r \in \mathbb{Z}_{+} \cdot$

Для приближения функции $h_{r, y}$ полиномами применяем лемму $9(n>2 s)$ :

$$
\left|h_{r, y}(x)-\frac{(x-y)^{r}}{2 r !}\left(p_{n, y}(x)-1\right)\right| \leqslant \frac{\gamma(s, t)}{2 r !} \Delta_{n}(x, y, s, t, r)
$$

Полагаем теперь при $N=n+r$

$$
p_{N}(x)=\int_{-1}^{1} \frac{(x-y)^{r}}{2 r !}\left(p_{n, y}(x)-1\right) d f^{(r)}(y)
$$

Так как все нули производной $p_{n, y}^{\prime}$, а значит, и всех следующих производных лежат на отрезке $[-1,1]$, то знаки $p_{n, y}^{(\nu)}(1), 0 \leqslant \nu \leqslant n$, совпадают со знаком старшего коэффициента $p_{n, y}$, который можно выбирать по своему желанию. 
Так как $r$ - нечетное число, то указанные полиномы приближают $h_{r, y}$ снизу. Учитывая убывание $f^{(r)}$, получаем, что $f(x) \leqslant p_{n}(x)$ для любого $x \in[-1,1]$. Имеем

$$
\int_{-1}^{1} \frac{p_{N}(x)-f(x)}{\left(1-x^{2}\right)^{\frac{r+1}{2}}} d x \leqslant \frac{\gamma(s, t)}{2 r !} \int_{-1}^{1} d f^{(r)}(y) \int_{-1}^{1} \frac{\Delta_{n}(x, y, s, t, r)}{\left(1-x^{2}\right)^{\frac{r+1}{2}}} d x .
$$

Выберем $s=\frac{r}{2}$ и $t=\frac{r+2}{2}$. Тогда

$$
\Delta_{n}(x, y, r / 2, r+2, r)=\left(\Delta_{n}(x, y, 1 / 2,1,1)\right)^{r} \Delta_{n}(x, y, 0,2,0) .
$$

Применяя к первому множителю лемму 10, а ко второму - лемму 11 , получаем

$$
\int_{-1}^{1} \frac{\Delta_{n}(x, y, r / 2, r+2, r)}{\left(1-x^{2}\right)^{\frac{r+1}{2}}} d x \leqslant \frac{3^{r}}{n^{r}} \int_{-1}^{1} \frac{\Delta_{n}(x, y, 0,2,0)}{\left(1-x^{2}\right)^{1 / 2}} d x \leqslant \frac{3^{r} c}{n^{r+1}} .
$$

При этом $n>2 s=r$, a $N=n+r \geqslant 2 r+1$.

ДоКАЗАТЕЛЬСТво ТЕОРЕМЫ 3 . Проведем рассуждения для отрезка $[-1,1]$ (замена $f(x)$ на $f(x-1)$ ). Тогда при $x \in[-1,1]$ имеем $f(x)=\int_{-1}^{1} h_{r, y}(x) d f^{(r)}(y)$ и, как и выше, при $N=n+r$ полагаем

$$
p_{N}(x)=\int_{-1}^{1} \frac{(x-y)^{r}}{2 r !}\left(p_{n, y}(x)-1\right) d f^{(r)}(y)
$$

где $p_{n, y}$ - полином из леммы 9 (любой из двух). Тогда $f(x) \leqslant p_{N}(x)$ при $x \in[-1,1]$ и все коэффициенты в разложении $p_{N}$ по степеням $(x-1)$ одного знака. Кроме того,

$$
\left|f(x)-p_{N}(x)\right| \leqslant \frac{\gamma(s, t)}{2 r !}\left|\int_{-1}^{1} \Delta_{n}(x, y, s, t, r) d f^{(r)}(y)\right|,
$$

где $s, t \in \mathbb{R}_{+}$определим позже, а $n>2 s$.

Чтобы оценить интеграл сверху, разобьем его на сумму интегралов по отрезкам $\left[y_{k}, y_{k+1}\right]$, где $y_{k}=\sin \frac{k \pi}{2 n+1}$ при $|k| \leqslant n$ и $y_{n+1}=-y_{-n-1}=1$. Очевидно, что если $\phi \in C[a, b]$ и $\phi(x) \geqslant 0$ при $x \in[a, b]$, а

$$
\max _{a \leqslant y \leqslant b} \phi(y) \leqslant \frac{c}{b-a} \int_{a}^{b} \phi(y) d y,
$$

то при любой возрастающей на $[a, b]$ функции $g$ имеем

$$
\int_{a}^{b} \phi(y) d g(y) \leqslant c \frac{g(b)-g(a)}{b-a} \int_{a}^{b} \phi(y) d y
$$

Именно таким свойством обладает подынтегральная функция $\Delta_{n}$ как функция от $y$ на каждом из отрезков $\left[y_{k}, y_{k+1}\right]$ при $k \in[-n, n-1]$ (см. лемму 12). Таким образом, имеем следующую оценку сверху интеграла в (11) (без окрестностей конщевых точек):

$$
\gamma_{1}(s, t, r) \sum_{k=-n}^{n-1} \frac{f^{(r)}\left(y_{k}\right)-f^{(r)}\left(y_{k+1}\right)}{y_{k+1}-y_{k}} \int_{y_{k}}^{y_{k+1}} \Delta_{n}(x, y, s, t, r) d y .
$$


Применяя лемму 3,1$)$ и свойство модуля $\omega$ (см. (5)), получаем неравенство

$$
\frac{f^{(r)}\left(y_{k}\right)-f^{(r)}\left(y_{k+1}\right)}{y_{k+1}-y_{k}} \leqslant \frac{\omega\left(f^{(r)} ; y_{k+1}-y_{k}\right)}{y_{k+1}-y_{k}} \leqslant 48 \frac{\omega\left(f^{(r)} ; \frac{\sqrt{1-y^{2}}}{24 n}\right)}{\frac{\sqrt{1-y^{2}}}{24 n}}
$$

и общую оценку

$$
\begin{gathered}
48 \gamma_{1}(s, t, r) \sum_{k=-n}^{n-1} \int_{y_{k}}^{y_{k+1}} \frac{n}{\sqrt{1-y^{2}}} \omega\left(f^{(r)} ; \frac{\sqrt{1-y^{2}}}{n}\right) \Delta_{n}(x, y, s, t, r) d y \\
\leqslant 48 \gamma_{1}(s, t, r) \int_{-1}^{1} \frac{n}{\sqrt{1-y^{2}}} \omega\left(f^{(r)} ; \frac{\sqrt{1-y^{2}}}{n}\right) \Delta_{n}(x, y, s, t, r) d y
\end{gathered}
$$

В силу неравенства (4) при $x \in(-1,1)$ имеем

$$
\omega\left(f^{(r)} ; \frac{\sqrt{1-y^{2}}}{n}\right) \leqslant \omega\left(f^{(r)} ; \frac{\sqrt{1-x^{2}}}{n}\right)\left(\frac{\sqrt{1-y^{2}}}{\sqrt{1-x^{2}}}+1\right) .
$$

Теперь нужно доказать, что при достаточно больших $s$ и $t$ вьполнено

$$
\int_{-1}^{1} \Delta_{n}(x, y, s, t, r)\left(\frac{n}{\sqrt{1-x^{2}}}+\frac{n}{\sqrt{1-y^{2}}}\right) d y \leqslant \gamma_{2}(s, t, r)\left(\frac{\sqrt{1-x^{2}}}{n}\right)^{r} .
$$

Выберем $s \geqslant \frac{r+1}{2}$ и $t=r+2$; тогда

$$
\Delta_{n}\left(x, y, \frac{r+1}{2}, r+2, r\right)=\left(\Delta_{n}\left(x, y, \frac{1}{2}, 1,1\right)\right)^{r} \Delta_{n}\left(x, y, \frac{1}{2}, 2,0\right) .
$$

Учитывая возрастание функции $\phi(t)=\frac{t}{t+a}, a>0$, на $\mathbb{R}_{+}$, к первому множителю можно применить лемму 10. Далее,

$$
\frac{\Delta_{n}(x, y, 1 / 2,2,0)}{\sqrt{1-x^{2}}} \leqslant \frac{\Delta_{n}(x, y, 0,2,0)}{\sqrt{1-y^{2}}}
$$

и в силу леммы 11

$$
\int_{-1}^{1} \frac{\Delta_{n}(x, y, 0,2,0)}{\sqrt{1-y^{2}}} d y=O\left(\frac{1}{n}\right) .
$$

Остается оценить в (11) интегралы по отрезкам $\left[-1,-y_{n}\right]$ и $\left[y_{n}, 1\right]$.

Очевидно, что $1-y_{n}^{2} \in\left[1 / 9 n^{2}, 1 / n^{2}\right]$, и в случае отрезка $\left[y_{n}, 1\right]$ имеем

$$
\begin{aligned}
& \left|\int_{y_{n}}^{1} \Delta_{n}\left(x, y, \frac{r+1}{2}, r+2, r\right) d f^{(r)}(y)\right| \\
& \quad \leqslant 3^{r}\left(\frac{\sqrt{1-x^{2}}}{n}\right)^{r}\left|\int_{y_{n}}^{1} \Delta_{n}\left(x, y, \frac{1}{2}, 2,0\right) d f^{(r)}(y)\right|
\end{aligned}
$$


При замене $x \in[-1,0]$ на $-x$ интеграл возрастает. Поэтому считаем далее $x \in$ $[0,1]$. Пусть сначала $x \in\left[0, y_{n}\right]$. Тогда

$$
\left|\int_{y_{n}}^{1} \Delta_{n}\left(x, y, \frac{1}{2}, 2,0\right) d f^{(r)}(y)\right| \leqslant\left|\int_{y_{n}}^{1} d f^{(r)}(y)\right| \leqslant \omega\left(f^{(r)} ; 1-y_{n}\right)
$$

и $1-y_{n} \leqslant \sqrt{1-x} \sqrt{1-y_{n}} \leqslant \frac{\sqrt{1-x}}{n}$.

Если же $x \in\left[y_{n}, 1\right]$, то имеем

$$
\left|\int_{y_{n}}^{1} \Delta_{n}\left(x, y, \frac{1}{2}, 0,0\right) d f^{(r)}(y)\right| \leqslant\left|\int_{y_{n}}^{1}\left(\frac{1-x^{2}}{1-x^{2}+1-y^{2}}\right)^{1 / 2} d f^{(r)}(y)\right|
$$

Применяя лемму 6 после замены $1-y=t$ при $a=1-x^{2}$ и $b=1-y_{n}$ и учитывая еще, что $1-x^{2} \leqslant 2(1-x) \leqslant 2\left(1-y_{n}\right)$, получаем

$$
\begin{aligned}
\int_{0}^{1-y_{n}}\left(\frac{1-x^{2}}{1-x^{2}+t}\right)^{1 / 2} d f^{(r)}(1-t) & \leqslant 5 \omega\left(f^{(r)} ; \sqrt{1-y_{n}} \sqrt{1-x^{2}}\right) \\
& \leqslant 5 \omega\left(f^{(r)} ; \frac{\sqrt{1-x^{2}}}{n}\right)
\end{aligned}
$$

При $\nu=0$ теорема 3 доказана.

Чтобы применить теперь лемму 4,1$)$ при $x_{1}=-1$ и $x_{2}=1$, проверим, что для построенного полинома $p_{N}$ имеют место равенства $p_{N}^{(\nu)}(-1)=f^{(\nu)}(-1), p_{N}^{(\nu)}(1)=$ $f^{(\nu)}(1)$ при $\nu \in[0, r]$.

Как видно из формулировки леммы 9 , при $y \in(-1,1)$ и $m<s$

$$
\frac{d^{m}}{d x^{m}}\left\{p_{n, y}(x)-1\right\}_{x=1}=\frac{d^{m}}{d x^{m}}\left\{p_{n, y}(x)-1\right\}_{x=-1}=0 .
$$

Поэтому $p_{n}^{(m)}(1)=0=f^{(m)}(1)$ при $m<s$ и $m \leqslant r$ (см. (10)). Выбираем $s=r+1$. Тогда при $m \leqslant r$ имеем

$$
\begin{aligned}
p_{N}^{(m)}(-1) & =\frac{1}{r !} \int_{-1}^{1} \frac{d^{m}}{d x^{m}}\left\{\frac{1}{2}(x-y)^{r}\left(p_{N, y}(x)+1\right)-(x-y)^{r}\right\}_{x=-1} d f^{(r)}(y) \\
& =\frac{(-1)^{r+1-m}}{(r-m) !} \int_{-1}^{1}(1+y)^{r-m} d f^{(r)}(y)=0=f^{(m)}(-1)
\end{aligned}
$$

(последнее равенство проверяется интегрированием по частям), $N=n+r>2 s+$ $r=3 r+2$. При $2 r+2 \leqslant n \leqslant 3 r+2$ для доказательства теоремы подходят два полинома из леммы 8 , интерполируюших $f$ в точках -1 и 0 с кратностью $r+1$ и с коэффициентами одного знака. 
ДОКАЗАТЕЛЬСТВО ТЕОРЕМЫ 4 . Сначала приблизим функцию $f$ сверху полиномами $q_{n}$ из $P_{n}(\mathbb{Z})$, а затем и из $P_{n}\left(\mathbb{Z}_{+}\right)$. В силу теоремы 3 , примененной к функции $f(x)-\sum_{k=0}^{r} \frac{f^{(k)}(0)}{k !} x^{k}$ на $[-1,0]$, при $n \geqslant 2 r+2$ сушествует полином $p_{n}$ такой, что $p_{n}^{(\nu)}(-1)=f^{(\nu)}(-1), p_{n}^{(\nu)}(0)=f^{(\nu)}(0), 0 \leqslant \nu \leqslant r$ и

$$
0 \leqslant p_{n}(x)-f(x) \leqslant \gamma_{0}(r) \delta_{0, n,-1,0}^{r}(x) \omega\left(f^{(r)} ; \delta_{0, n,-1,0}(x)\right) .
$$

Перейдем к отрезку $[0,1]$ заменой $x$ на $-x$. По условию теоремы теперь $\frac{f^{(\nu)}(0)}{\nu !}$ и $\frac{f^{(\nu)}(1)}{\nu !} \in \mathbb{Z}, 0 \leqslant \nu \leqslant r$, и, значит, эрмитовский полином $h$, определяемый условиями $h^{(\nu)}(0)=f^{(\nu)}(0), h^{(\nu)}(1)=f^{(\nu)}(1), 0 \leqslant \nu \leqslant r$ имеет целые коэффициенты.

Полагаем $X(x)=x(1-x)$. На отрезке $[0,1]$ имеем $0 \leqslant X(x) \leqslant 1 / 4$. Учитывая, что $\left(p_{n}-h\right)^{(\nu)}(x)=0$ при $x=0, x=1, \nu \in[0, r]$, разложим полином $h-p_{n}$ по степеням полинома $X$ :

$$
\left(h-p_{n}\right)(x)=\sum_{k=r+1}^{N_{1}}\left(a_{k} x+b_{k}(1-x)\right) X^{k}(x), \quad N_{1}=\left[\frac{n}{2}\right] .
$$

Применим лемму 13 при $r+1 \leqslant k<k+m=3 r+3$, заменяя $x$ на $X$ при $x \in[0,1]$, $\{c\}=c-[c] \in[0,1)$. Имеем

$0 \leqslant\left(\left\{a_{k}\right\} x+\left\{b_{k}\right\}(1-x)\right)\left(X^{k}(x)-X^{3 r+3}(x) s(X(x))\right) \leqslant \gamma_{1}(r) \min \left\{\frac{1}{n^{2 k}}, X^{k}(x)\right\}$.

Поэтому при некоторых новых коэффициентах

$$
\begin{aligned}
0 & \leqslant h(x)-p_{n}(x)-\sum_{k=r+1}^{3 r+2}\left(\left[a_{k}\right] x+\left[b_{k}\right](1-x)\right) X^{k}(x) \\
& \leqslant \sum_{k=3 r+3}^{N_{1}}\left(a_{1, k} x+b_{1, k}(1-x)\right) X^{k}(x)+\gamma_{1}(r) \min \left\{\frac{1}{n^{2 r+2}}, X^{r+1}(x)\right\} .
\end{aligned}
$$

Вынося из суммы множитель $X^{r+1}$ (при $N_{1}<3 r+3$ суммы нет) и переразлагая полином, получаем $\left(N_{2}=N_{1}-r-1\right)$

$$
X^{r+1}(x) \sum_{k=2 r+2}^{N_{2}}\left(c_{k} x+d_{k}(1-x)\right) X^{k}(x)(1-X(x))^{N_{2}-k} .
$$

Но тогда

$$
\begin{aligned}
0 \leqslant & h(x)-p_{n}(x)-\sum_{k=r+1}^{3 r+2}\left(\left[a_{k}\right] x+\left[b_{k}\right](1-x)\right) X^{k}(x) \\
& -X^{r+1}(x) \sum_{k=2 r+2}^{N_{2}}\left(\left[c_{k}\right] x+\left[d_{k}\right](1-x)\right) X^{k}(x)(1-X(x))^{N_{2}-k} \\
& \leqslant \gamma_{1}(r) \min \left\{\frac{1}{n^{2 r+2}}, X^{r+1}(x)\right\}+X^{r+1}(x) \sum_{k=2 r+2}^{N_{2}} X^{k}(x)(1-X(x))^{N_{2}-k}
\end{aligned}
$$


Второе слагаемое не больше

$$
\begin{gathered}
X^{r+1}(x)\left(\sum_{k=2 r+2}^{N_{2}-2 r-2} X^{k}(x)(1-X(x))^{N_{2}-k} \frac{\left(\begin{array}{c}
N_{2} \\
k
\end{array}\right)}{\left(\begin{array}{c}
N_{2} \\
2 r+2
\end{array}\right)}+\sum_{k=N_{2}-2 r-1}^{\infty} X^{k}(x)\right) \\
\leqslant X^{r+1}(x)\left(\frac{1}{\left(\begin{array}{c}
N_{2} \\
2 r+2
\end{array}\right)}+\frac{4}{3}\left(\frac{1}{4}\right)^{N_{2}-2 r-1}\right) \\
\leqslant \gamma_{2}(r)\left(\frac{X(x)}{n^{2}}\right)^{r+1} \leqslant \gamma_{2}(r) \min \left\{\frac{1}{n^{2 r+2}}, X^{r+1}(x)\right\} .
\end{gathered}
$$

Таким образом, построен полином $q_{n} \in P_{n}(\mathbb{Z})$ такой, что на отрезке $[0,1]$

$$
0 \leqslant q_{n}(x)-p_{n}(x) \leqslant \gamma_{3}(r) \min \left\{\frac{1}{n^{2 r+2}}, X^{r+1}(x)\right\}
$$

и $q_{n}^{(\nu)}(x)=p_{n}^{(\nu)}(x)$ при $x=0, x=1, \nu \in[0, r]$.

В силу леммы 4,2$)$ при $x \in[0,1]$ и $\nu \in[0, r]$ имеем

$$
\left|p_{n}^{(\nu)}(x)-q_{n}^{(\nu)}(x)\right| \leqslant \gamma_{4}(r) \min \left\{\frac{1}{n^{2(r+1-\nu)}}, X^{r+1-\nu}(x)\right\} .
$$

Из соотношений (12) и (13) получаем

$$
\begin{aligned}
0 & \leqslant q_{n}(x)-f(x) \\
& \leqslant \gamma_{5}(r)\left(\delta_{0, n, 0,1}^{r}(x) \omega\left(f^{(r)} ; \delta_{0, n, 0,1}(x)\right)+\min \left\{\frac{1}{n^{2(r+1)}}, X^{r+1}(x)\right\}\right),
\end{aligned}
$$

а в силу леммы 1 , леммы 4,1$)$ и неравенства (14) имеем при $1 \leqslant \nu \leqslant r$

$$
\begin{aligned}
& \left|f^{(\nu)}(x)-q_{n}^{(\nu)}(x)\right| \\
& \quad \leqslant \gamma_{6}(r)\left(\delta_{0, n, 0,1}^{r-\nu}(x) \omega\left(f^{(r)} ; \delta_{0, n, 0,1}(x)\right)+\min \left\{\frac{1}{n^{2(r+1-\nu)}}, X^{r+1-\nu}(x)\right\}\right)
\end{aligned}
$$

и

$$
\left|q_{n}^{r+1}(x)\right| \leqslant \gamma_{7}(r)\left(\frac{\omega\left(f^{(r)} ; \delta_{n, 0,1}(x)\right)}{\delta_{n, 0,1}(x)}+1\right) .
$$

Теперь вернемся к отрезку $[-1,0]$ и приблизим $q_{n}(-x)$ сверху полиномами из $P_{n}\left(\mathbb{Z}_{+}\right)$. В силу $(17)$ и $(5)$ при $x \in[-1,0]$ имеем

$$
\left|q_{n}^{r+1}(-x)\right| \leqslant \gamma_{8}(r)\left(n^{2} \omega\left(f^{(r)} ; \frac{1}{n^{2}}\right)+1\right) .
$$

Но тогда в силу неравенства В.А. Маркова (см., например, [1, п. 4.8.8]) при $\nu \in$ $[0, n-r-1]$ получаем

$$
\left|q_{n}^{(r+1+\nu)}(0)\right| \leqslant \gamma_{8}(r)\left(n^{2} \omega\left(f^{(r)} ; \frac{1}{n^{2}}\right)+1\right) 2^{\nu} T_{n-r-1}^{(\nu)}(1),
$$


где $T_{n}(x)=\cos n \arccos x$.

Заменим в неравенствах (15) и (16), где вместо $x$ можно поставить $-x \in[0,1]$, $q_{n}(-x)$ на

$$
\begin{aligned}
& q_{n}(-x)+\left(\gamma_{8}(r)\left(n^{2} \omega\left(f^{(r)} ; \frac{1}{n^{2}}\right)+1\right)+1\right) \\
& \times\left(T_{n}(2 x+1)+1\right)\left(x+x^{2} q_{n}^{+}(x)\right)^{r+1}
\end{aligned}
$$

здесь $q_{n}^{+}$- полином из леммы 14 . Так как $T_{n}(2 x+1)+1 \geqslant 0$ при $x \in[-1,0]$, а $r-$ нечетное, то график этого полинома выше графика $q_{n}(-x)$, а все его коэффициенты целые, поскольку таковыми являются коэффициенты $q_{n}^{+}$и $T_{n}(x+1)$.

Проверим, что все коэффициенты этого полинома положительные. При $\nu \in$ $[0, r]$ нужно лишь учесть, что $q_{n}^{(\nu)}(0)=f^{(\nu)}(0)$ и воспользоваться условием теоремы. Так как $T_{n}^{(\nu)}(1)$ возрастает вместе с $\nu$ на $[0, n]$ и $n$, то при $\nu \in[0, n-r-1]$ в силу (18) имеем

$$
\begin{aligned}
\left|q_{n}^{(r+1+\nu)}(0)\right| & \leqslant \gamma_{8}(r)\left(n^{2} \omega\left(f^{(r)} ; \frac{1}{n^{2}}\right)+1\right) 2^{\nu} T_{n}^{(\nu+r+1)}(1) \\
& =\gamma_{8}(r)\left(n^{2} \omega\left(f^{(r)} ; \frac{1}{n^{2}}\right)+1\right) \frac{d^{\nu+r+1}}{d x^{\nu+r+1}}\left\{T_{n}(2 x+1)\right\}_{x=0} 2^{-r-1}
\end{aligned}
$$

а поскольку коэффициенты $q_{n}^{+}$положительные, то и все коэффициенты указанного полинома (степени не вьше $(n+2)(r+1)+n)$ положительные.

Разность между производными порядка $\nu \in[0, r]$ функции и этого полинома по модулю (см. (16), (19) и леммы 14 и 4,2$))$ не больше

$$
\begin{aligned}
& \gamma_{9}(r)\left(\delta_{0, n,-1,0}^{r-\nu}(x) \omega\left(f^{(r)} ; \delta_{0, n,-1,0}(x)\right)+\min \left\{\frac{1}{n^{2(r+1-\nu)}},(|x|(x+1))^{r+1-\nu}\right\}\right) \\
& +\left(n^{2} \omega\left(f^{(r)} ; \frac{1}{n^{2}}\right)+1\right) \min \left\{\frac{1}{n^{2(r+1-\nu)}},(|x|(x+1))^{r+1-\nu}\right\} .
\end{aligned}
$$

В силу (5) при $\delta \geqslant 0$ и $h \geqslant 0$

$$
n^{2} \omega\left(\frac{1}{n^{2}}\right) \min \left\{\frac{1}{n^{2}}, h\right\}^{1+\delta} \leqslant 2 \omega\left(\min \left\{\frac{1}{n^{2}}, h\right\}\right) \min \left\{\frac{1}{n^{2}}, h\right\}^{\delta},
$$

a

$$
\min \left\{\frac{1}{n^{2}},|x|(x+1)\right\} \leqslant \min \left\{|x|(x+1), \frac{\sqrt{|x|(x+1)}}{n}\right\} \leqslant 2 \delta_{0, n,-1,0}(x) .
$$

Теорема 4 доказана для степеней $n \geqslant n_{0}(r)$, а при $2 r+2 \leqslant n<n_{0}(r)$ можно взять интерполяционньй полином $h_{2 r+2}$ из леммы 8,2$)$.

Отметим еще, что функцию $f_{1}(x)=x / 2$ на любом отрезке $[-\delta, \delta], \delta>0$, нельзя приблизить с одной стороны полиномами с целыми коэффициентами, а для функции $f_{2}(x)=x^{3}$ вообще не существует полинома $p_{4}$ с отрицательными коэффициентами и условиями $f_{2}^{(\nu)}(-1)=p_{4}^{(\nu)}(-1), f_{2}^{(\nu)}(0)=p_{4}^{(\nu)}(0), \nu=0,1$, и $p_{4}(x) \geqslant f_{2}(x)$ на $[-1,0]$. 


\section{Список литературы}

1. Тиман A. Ф. Теория приближения функций действительного переменного. М.: Наука, 1960.

2. Дзядык B. K. Введение в теорию равномерного приближения функций полиномами. М.: Наука, 1977.

3. Гопенгауз И. E. К теореме А. $\Phi$. Тимана о приближении функций многочленами на конечном отрезке // Матем. заметки. 1967. Т. 1. № 2. С. 163-172.

4. Gopengauz I. E. Pointwise Estimates of the Hermitian Interpolation // J. Appr. Theory. 1994. V. 77. № 1. P. 31-41.

5. Brudnyi Yu. A. Timans type result on approximation by algebraic polynomials // Operator Theory. Advances and Applications. 1997. V. 98. P. 92-101.

6. Суетин П. К. Классические ортогональные многочлены. М.: Наука, 1979.

7. Hewitt E., Zuckerman H. Approximation by polynomials with integral coefficients a reformulation of the Stone-Weierstrass theorem // Duke Math. J. 1959. V. 26. P. 305-324.

8. Гельфонд А. О. О равномерных приближениях многочленами с целыми коэффициентами // УМН. 1955. Т. 10. № 1. С. 41-65.

9. Тригуб Р. М. Приближение функций многочленами с целыми коэффициентами // Изв.АН CССР. Сер. матем. 1962. T. 26. № 2. С. 261-280.

10. Тригуб Р. М. Приближение функций с диофантовыми условиями многочленами с целыми коэффициентами // Метрические вопросы теории функций и отображений. № 2. Киев: Наукова думка, 1971. С. 267-333.

11. Ferguson B. O. Approximation by Polynomials with Integral Coefficients // Math. Surveys. № 17. Providence, R. I.: Amer. Math. Soc., 1980.

12. Субханкулов М. А. Тауберовы теоремы с остатком. М.: Наука, 1976.

13. Toland J. E. Selp-adjoint operators and cones // J. London Math. Soc. 1996. V. 53. № 2. P. $167-183$.

14. Тригуб Р. М. Односторонние и комонотонные приближения гладких функций алгебраическими полиномами с положительными коэффициентами // Докл. РАН. 2000. Т. 370. № 2. C. 168-170.

15. Тригуб Р. М. Теорія наближення функцій та іі застосування // Праці Інституту матем. НАН Украіни. Т. 31. Киів: Ін-т матем. НАН Украіни, 2000. С. 461-476.

16. Тригуб Р. М. Приближение индикатора интервала алгебраическими полиномами с эрмитовской интерполяцией в двух точках // Тр. Ин-та прикл. мат. и мех. Т. 4. Донецк, 1999. С. $186-194$. 